\title{
Sensibilización y disposición al cambio, a la luz de los retos y desafíos de la actual coyuntura educativa
}

\section{Sensitization and Disposition to Change Facing the Challenges of the New Educational Circumstances}

\author{
Luis Alfredo Miranda Calderón ${ }^{l}$ \\ División de Educación Básica \\ Centro de Investigación y Docencia en Educación (CIDE) \\ Universidad Nacional de Costa Rica \\ Heredia, Costa Rica \\ alfremira@gmail.com
}

Kenneth Cubillo Jiménez ${ }^{2}$

División de Educación Rural

Centro de Investigación y Docencia en Educación (CIDE)

Universidad Nacional de Costa Rica

Heredia, Costa Rica

kecuji@gmail.com

Recibido 04 de agosto de $2010 \bullet$ Aceptado 31 de agosto de 2010

Resumen. El artículo aborda la necesidad de una renovada visión de la formación docente a partir de los últimos estudios y avances en torno al tema del aprendizaje de las personas a lo largo de sus vidas. Expone un conjunto de temáticas inspiradas en los aportes de nuevos campos de estudio vinculados al aprendizaje, tal es el caso de la neurociencia. Estas deben ser consideradas en las decisiones que orienten la formación de las futuras y futuros docentes y así propiciar la transformación en la práctica pedagógica. Se plantea, como condición imprescindible, la sensibilización y disposición al cambio desde los formadores de docentes, para detonar procesos de investigación y generación de propuestas pedagógicas, en concordancia con la nueva y dinámica sociedad del conocimiento.

Palabras clave. Aprendizaje, formación de formadores, neurociencia, transformación pedagógica.

\footnotetext{
Bachiller en Artes Dramáticas, Bachiller en Ciencias de la Educación en I y II Ciclos, Licenciado en Educación Rural, Magíster con especialidades en Administración Educativa y en Formación de Formadores de la Educación Básica. Maestro rural durante dieciocho años en una comunidad rural costera, donde ha realizado diversas investigaciones y exploraciones relacionadas con el aprendizaje mediado por uso y aprovechamiento del entorno natural. Maestro en la Escuela Nueva Laboratorio de la Universidad de Costa Rica donde puso en práctica y sistematizó procesos pedagógicos mediados por el teatro como vehículo para el aprendizaje significativo. Desde 2005 se ha desempeñado como docente en la Universidad de Costa Rica y, posteriormente, como académico e investigador en la División de Educación Rural de la Universidad Nacional de Costa Rica. Actualmente labora como académico en la División de Educación Básica de la Universidad Nacional. Asimismo conforma, en la División de Educación Rural, la comisión responsable del Diseño de la Licenciatura en Educación Secundaria Rural.

2 Licenciado en Educación con énfasis en Educación Rural I y II Ciclos, Magister en Formación de Formadores de Educación General Básica. Maestro de educación primaria en áreas básicas, religión y artes. Actualmente se desempeña como docente, investigador y extensionista de la División de Educación Rural de la Universidad Nacional de Costa Rica, en donde es coordinador de carrera en Limón y en el marco del Convenio CONARE-UNA-UCR. Responsable del proyecto: "Las tecnologías para la información y la comunicación como complemento para el fortalecimiento de los procesos de formación inicial, continua y permanente de la División de Educación Rural”.
} 
Abstract. This article is about the necessity of an up to date vision in teachers' training. It starts with the latest studies and advances surrounding learning-along-life term. It presents a set of topics inspired in the contributions of new fields of study connected to learning. Such contributions, as neuroscience, should be considered in the decisions related to future teachers' training, thus favoring the transformation of the pedagogical practice. Awareness and disposition to change are essential requirements for teacher trainers who will drive investigative processes and pedagogical proposals generation; all of this according to the new and dynamic knowledge society.

Key word. Teacher trainers, neuroscience, pedagogical transformation.

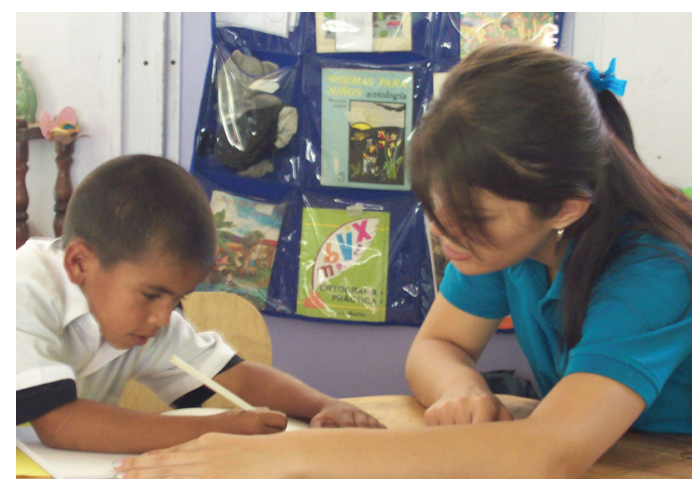

\author{
"Cualquier acción que intente \\ hacerle ver otras cosas al maestro, \\ tiene que empezar por derrumbar \\ lo que él suponía bien establecido y clasificado"
}

Emilia Ferreiro

La Educación, en el marco de la post-modernidad, adquiere carácter trascendental en la construcción del destino de la humanidad y de las naciones. Vista no solamente como un fenómeno social, sino además como indicador fiel de la evolución del quehacer humano, la educación constituye una de las disciplinas que sugieren y marcan pautas en la toma de decisiones de los gobiernos y de los grupos de poder que prevalecen en toda sociedad.

En cada una de las sociedades que han elegido la educación como herramienta para alcanzar el desarrollo, se le ha valorado como una actividad mediante la cual se produce y distribuye el conocimiento y no como un proceso encaminado hacia la obtención de logros en el futuro. Se desvirtúa así uno de los propósitos primordiales de la educación: conceder respuestas anticipadas a las situaciones que depara la sociedad cambiante. Los nuevos tiempos nos sitúan hoy ante un acelerado proceso de cambios que ha modificado la forma en que estaba organizado el mundo. Cambios que inevitablemente inciden en la educación y que nos obligan a transformar, de forma dinámica, prácticas, teorías, modelos pedagógicos y contenidos. Pero sobre todo, nuestros supuestos epistemológicos y ontológicos como pedagogos y a la luz de una nueva sociedad del conocimiento.

Las organizaciones educativas en cualquiera de sus modalidades, de ser organizaciones sencillas y de fácil manejo, han pasado a ser organizaciones más complejas con personal calificado y con diversidad de funciones. A esto se le debe agregar el conjunto de fenómenos sociales que inciden directa o indirectamente en la sociedad, tal es el caso del fenómeno demográfico de la inmigración, el crecimiento acelerado de las concentraciones de población y la mayor cobertura que debe asumir el sistema educativo. Estas condiciones, han transformado no solamente la realidad educativa y ampliado el horizonte social que la educación debe abarcar, sino que, en consecuencia, plantean nuevos retos y desafíos a las instancias formadoras de los futuros y futuras docentes. 


\section{Nuevos tiempos, nuevos retos}

Frente a la actual coyuntura histórica de la educación, desde muchos sectores se plantea la necesidad de introducir concepciones innovadoras en los sistemas de formación docente. Y esto no sólo en función de la calidad de la educación, sino también en función de la necesidad de dotar al país de mecanismos que detonen e impulsen el desarrollo. Sin embargo, los desafíos que nos plantea el nuevo milenio revisten de complejidad esta actividad por lo que dichos retos debieran visualizarse como oportunidades de cambio más que como obstáculos sin solución.

Entre los nuevos desafíos, producto de esta coyuntura histórica se mencionan los siguientes, detectados por Coronado, Goñi, Meza, Vargas y Francis (2009), en el marco del proyecto de CONARE denominado: Actualización Pedagógica de Formadores de las Carreras de Educación de las Universidades Estatales.

- Poca concordancia e inexistencia de políticas que orienten la formación profesional de los docentes, eso evidencia en la desarticulación del sistema educativo respecto de la formación docente: universidad-Ministerio de Educación Pública-sociedad y el poco seguimiento de las prácticas formativas con respecto a la visión de Estado que se define para el futuro del país.

- Desconocimiento de las necesidades y el contexto cultural de los estudiantes que integran los programas de formación docente.

- Procedimientos de selección y admisión de los estudiantes que no permiten reconocer un perfil de ingreso más adecuado.

- Los formadores presentan un déficit en las bases teórico-pedagógica. No existe una formación ni una postura epistemológica, se distingue una ausencia de criticidad del propio paradigma epistemológico que más se ajusta a disciplina de la praxis pedagógica.

- Descontextualización y falta de vivencias en la formación didáctica que se ajuste a cada disciplina. Se carece de formación en el área de docencia universitaria y en el uso de tecnologías de la información y comunicación como herramientas para la formación profesional de docentes.

- Desconocimiento de las implicaciones éticas políticas del paradigma epistemológico que fundamente el ser y el quehacer del docente. Existe una descontextualización de las fuentes teóricas, existe una réplica acrítica de textos, se promueven prácticas más conductistas que constructivistas.

- No existe trabajo conjunto y colaborativo entre las universidades estatales.

- Existe una desvalorización de la esencia del trabajo académico universitario como fuente fundamental de la práctica de la formación de docentes: la investigación como fuente de la docencia. (p. 7)

Ante estos condicionantes, las instancias estatales formadoras de formadores deberían brindar una respuesta oportuna en términos de impacto y pertinencia, no solo en la formación de los futuros docentes, sino en las decisiones que atañen al diseño y cumplimiento de políticas educativas que tengan incidencia pertinente en el contexto educativo costarricense. En este sentido, es necesario destacar el papel de los movimientos de renovación y contextualización de la oferta académica en el campo de la pedagogía, los cuales en las últimas décadas han impulsado las facultades que atienden la formación docente en cada una de las universidades públicas; pero que desafortunadamente no han permeado la cotidianidad del aula en cualquiera de sus niveles o modalidades. 
La discusión sobre la formación de formadores docentes en el marco de las universidades estatales responde a una temática compleja, porque implica discutir implícitamente la multiplicidad de variables que intervienen en la organización universitaria, sin perder de vista las limitaciones generadas por su desarticulación con la instancia estatal de educación. Esta situación de divorcio entre las instancias formadoras de docentes y el ente empleador apunta a futuros problemas, en caso de no abordarse las decisiones necesarias para atender la formación de los futuros docentes, en correspondencia con el campo de acción en el que se eventualmente deberán desempeñarse, así como la capacitación para su ejercicio profesional, los modelos pedagógicos y de gestión de la educación pública, en general, y también en lo particular.

El carácter poco sistemático y desarticulado de las políticas de la formación docente entre las universidades y con el ente empleador del profesorado no ha permitido encarar, hasta ahora, el problema desde una perspectiva más coherente y unificada. Por lo general, se ha tratado de llevar a la práctica estrategias apoyadas en metodologías obsoletas, las cuales, en definitiva, no han ayudado a solucionar los problemas que continuamente plantea la educación.

Todavía hace treinta años, los educadores y educadoras desconocían los aportes de los científicos cognitivos; y los investigadores en el naciente campo de la ciencia cognitiva desarrollaban sus investigaciones en forma teórica, alejados de la realidad de las aulas. Hoy, los investigadores cognitivos dedican más tiempo al trabajo al lado de maestros y maestras, someten sus teorías a pruebas en aulas reales, donde pueden observar cómo diferentes escenarios e interacciones en el salón de clases influyen en las aplicaciones de sus teorías. De esta manera, la posibilidad de consolidar conocimiento útil y veraz concede la esperanza de mejorar paulatinamente las prácticas en la formación de formadores y formadoras, en el devenir de la evolución educativa.

Emprender una expedición hacia la búsqueda, aplicación y reconstrucción de este nuevo conocimiento sobre el aprendizaje, desde la formación docente, nos sitúa a muchos y a muchas ante una nueva lectura de nuestro rol en dicha formación. El conocimiento debe ser concebido desde nuevos paradigmas y posiciones metodológicas que aborden no solamente la especificidad de la docencia, sino sus implicaciones como factor determinante en el sistema educativo y como punto de partida para una renovada visión de la formación docente, donde los formadores de formadores seamos protagonistas y aprendices. Descubrir y potenciar, desde la metacognición del grupo docente, la parte atractiva del conocimiento, la faceta que detona el interés e incide en nuestro gusto hacia lo nuevo, lo imaginario y lo que alimenta áreas perceptivas más importantes de nuestro ser. Una nueva perspectiva que nos conceda la oportunidad de reflexionar acerca de nuestra labor formativa y el requerido aporte a una educación en constante evolución, como lo menciona Latapí (2003):

(...) la formación de los maestros no es solo es asunto central para mejorar la educación sino constituye el mecanismo central para reoxigenar sistema educativo: los nuevos maestros no solo son sustitutos de los que mueren o se jubilan, son la vía por la que el sistema renueva sus prácticas, cuestiona sus tradiciones, acepta nuevas visiones teóricas, se abre al conocimiento y se revitaliza. (p. 7)

Comprender la educación como un quehacer intrínseco de la sociedad, que si bien sirve a los fines planteados por esta para perpetuar o transformar la civilización y todo lo que esto implica, es entender que la educación debe alimentarse y resurgir de su contexto, considerando e interpretando su entorno más inmediato: los seres humanos y el funcionamiento de su mente.

Desde esta visión, la formación docente debe marcar el rumbo en la concepción de aportes, no solo desde la retórica y el discurso académico, sino aplicativos y en función de su incidencia en 
la pedagogía contemporánea, ahora más enriquecida por los aportes científicos mencionados. Por lo tanto, de alguna manera, el aprendizaje y la adquisición de conocimiento, desde esta óptica, dependen en gran medida del comportamiento y actitudes de los futuros y futuras docentes, de la metodología que utilizarán y de las distintas formas de interacción posibles que logren generar en el aula escolar.

Por otra parte y a partir de una renovada visión de las escuelas de educación de las universidades estatales, se hace impostergable una detalla revisión de sus planes de estudio, mallas curriculares, perfiles de los formadores y graduados, así como sus implicaciones en el orden educativo actual. Al respecto, los centros de formación de docentes requieren homogenizar y uniformar el discurso pedagógico que defina los parámetros mínimos que deben ser entendidos no solo en el ínterin universitario, sino además con una fuerte intervención en el sistema formal de enseñanza. De lo contrario, el conocimiento que construyamos en nuestras instancias formadoras resultaría indiferente en el devenir educativo de esta nación.

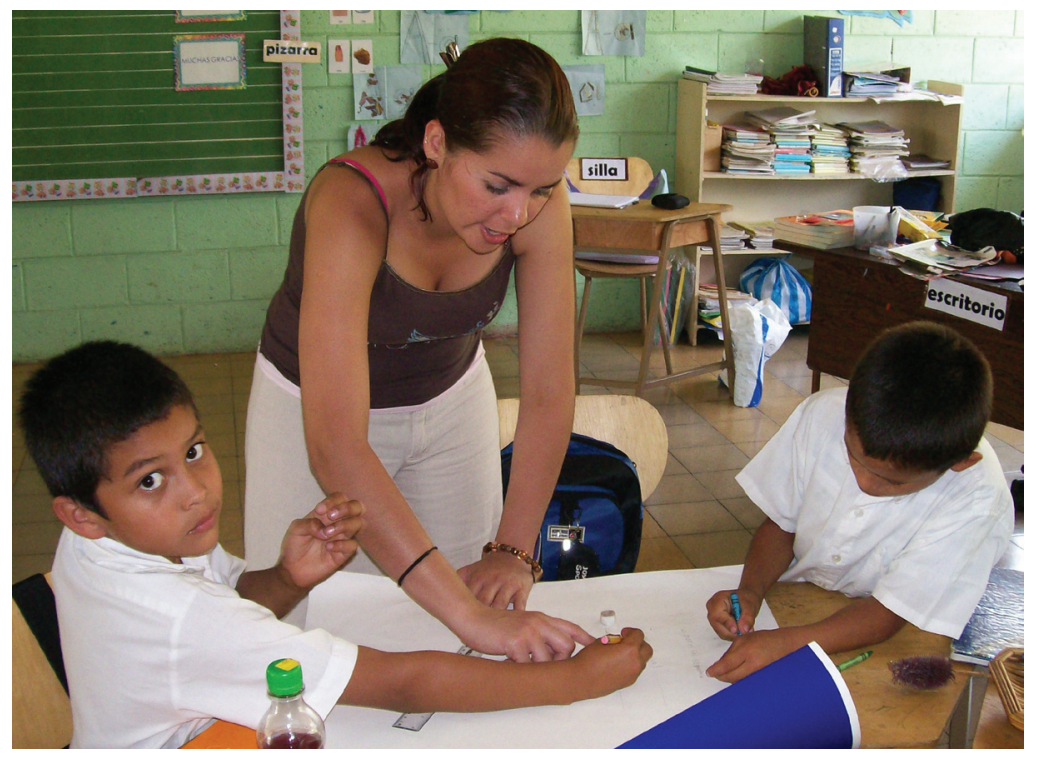

Una acción formativa docente pertinente y eficaz para responder a las exigencias del contexto nacional actual

Las exigencias y demandas de la dinámica sociedad costarricense nos están indicando, desde hace ya algunas décadas, la necesidad imperante de conectar la teoría con la práctica, no solo en el discurso educativo, sino también en todo los campos que intervienen en la evolución social de esta nación. Las importantes cifras mostradas en diversos estudios corroboran, una vez más, la existencia de múltiples factores que inciden en la condición actual de la educación y otros servicios que el Estado debe garantizar a sus pobladores, tal es el caso de los fenómenos demográficos, provocados por la migración como producto directo de los procesos globalizantes, los cuales, de forma inevitable y contundente, afectan los procesos educativos. Para ejemplificar, es necesario mencionar la conformación de comunidades y poblaciones culturalmente diversas, como una condición real reflejada y cada vez más latente en las aulas de preescolar primaria y secundaria a lo largo del país. Esta realidad debe ser asumida como compromiso esencial por las escuelas de formación docente, para proyectarse, en el devenir educativo, desde una formación adecuada y pertinente, acorde con la cada vez más compleja sociedad costarricense. 
Si hablamos de pertinencia en la formación docente, debemos comprender que la misma debe estar ligada necesariamente a lo que sucede o no sucede en el aula escolar. Los cambios que ha experimentado y experimentará "la escuela", no solo deben ser precisados y atendidos por la instancia gubernamental de educación, sino que deben constituirse en la materia prima de investigación y generación de propuestas, en concordancia con el actual horizonte educativo en el que, eventualmente, nuestros estudiantes-docentes deberán desempeñarse. Ninguna política de formación docente puede encontrar asidero real sin determinar, con precisión, la realidad a la que eventualmente los jóvenes y las jóvenes docentes deberán enfrentar y transformar y el amplio espectro de modalidades y ofertas educativas que impera en el contexto nacional. Del mismo modo, para establecer criterios para el diseño de nueva oferta curricular en la formación de una nueva generación de docentes, es necesario generar nuevo conocimiento que aporte información actual de las condiciones reales de las comunidades donde están insertas dichas instituciones educativas. Es necesario, además, hacer una adecuación del sistema de formación de docentes y del compromiso que tienen el sistema educativo y las instituciones que forman profesores, para poder cumplir con la cantidad y calidad de personal que el sistema educativo requiere para hacer frente a los retos que plantea la educación en los distintos niveles y modalidades.

Un tema importante en relación con los desafíos a encarar es, precisamente, la necesidad de acuerdos del reordenamiento de la oferta de formación docente dentro de las distintas regiones del país; problemática que no solamente sobrepasa la mera mirada institucional, sino que compromete a diversas instancias y sectores de la comunidad costarricense beneficiadas directa o indirectamente de la actividad educativa.

\section{Las nuevas expectativas de los futuros docentes}

Nuestras experiencias en la formación de educadoras y educadores nos han mostrado, repetidas veces, que gracias al conocimiento, basado primero en la experiencia acerca de las expectativas y demandas reales de aprendizaje de los futuros docentes, unido al desarrollo de la sensibilidad hacia la forma más adecuada que atienda la complejidad de la formación de formadores, los docentes universitarios debemos generar procesos investigativos que aporten conocimiento útil atinente a nuestra práctica educativa. El estudio de las expectativas de aprendizaje de nuestros estudiantes podría aportar información estratégica para mejorar y fortalecer la formación docente en momentos en que resulta indispensable una profunda revisión de los modelos educativos vigentes. Por lo tanto, se podría afirmar que la valoración de las expectativas de docentes en formación podría constituirse en un excelente indicio para el establecimiento de planes de estudio, ofertas académicas y propuestas de investigación para estudios situados en este nuevo ámbito o temática.

El formador de formadores, por su carácter inspirador, debe brindar opciones para generar este tipo de procesos de investigación, en las mismas facultades y centros de formación para la docencia, pues el mejor componente que puede orientar la práctica educativa es el conglomerado de expectativas de quienes aprenden. Sin embargo, debemos tener claro que tales expectativas de la formación de docentes, como propone Pichardo (2007, p. 4), se orientan en dos vías: “(...) aquellos que investigan las expectativas del alumnado con la intención de conocer qué esperan de la universidad en general, y aquellos que estudian las expectativas de los estudiantes sobre componentes específicos del proceso de enseñanza-aprendizaje". 
Desde este enfoque, el rol docente, se presenta, muchas veces, descontextualizado y encadenado a una concepción curricular rígida y dominante, así como a la lógica de las relaciones docenteestudiante que le son propias. Por tanto, se recarga el trabajo en el profesorado y se incrementa la tensión entre los intereses de estudiantiles y la posición de docente. En este marco situacional, pasamos por alto, generalmente, cómo conciben nuestros estudiantes su propia formación; lo que esperan de la academia y cómo se visualizan en su práctica profesional. Esta disposición a la reflexión basada en las expectativas de ambos actores del acto formativo se hace necesaria como factor y precondición del cambio y la innovación; pero, además, se deben introducir elementos de análisis de la realidad, así como de crítica social; puesto que se persigue concebir, al nuevo docente, como reformador social o agente de cambio. Si esto no ocurre, solo se van a generar más docentes incapaces de asumir tal reto y muchos estudiantes de educación que pasan de un tipo de experiencia

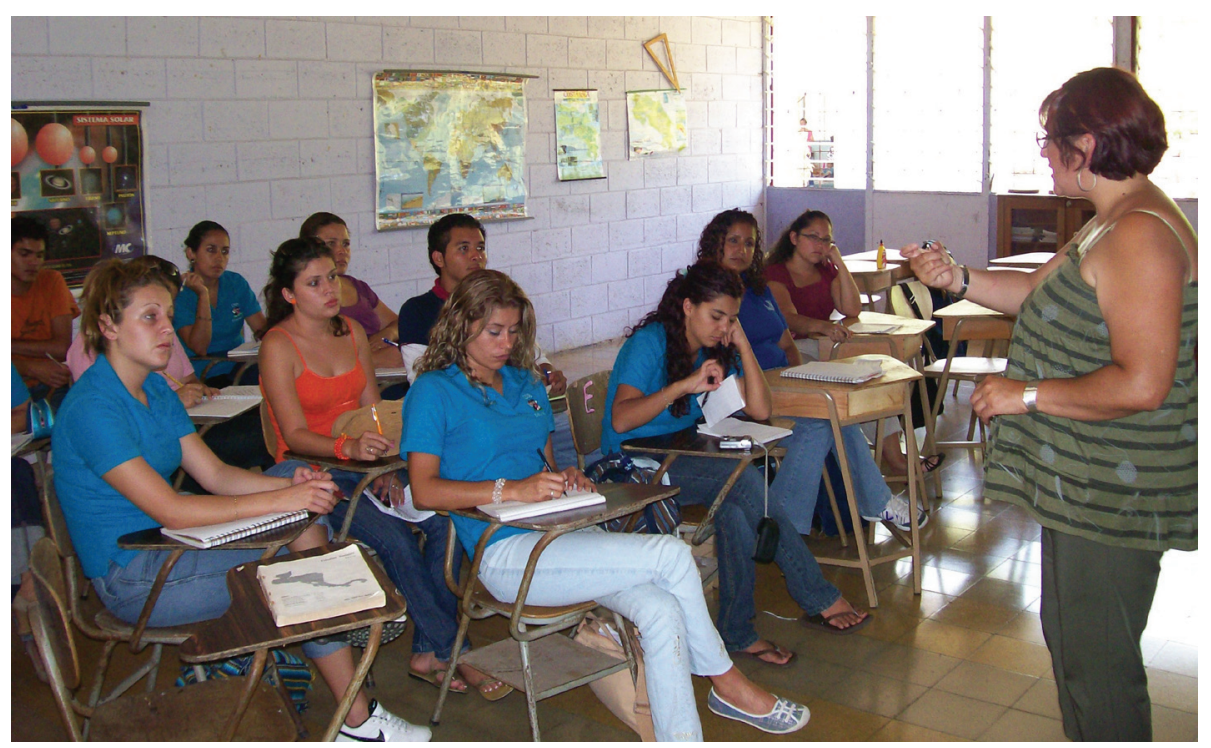

de formación tradicional a otra "innovadora" o "renovada", sin tener muy claro de qué se trata en cada caso.

\section{Los nuevos aportes de la neurociencia: itinerario para el cambio desde una nueva concepción de la formación de formadores}

Las incertidumbres han venido sugiriendo, desde hace décadas y desde los diversos campos que atienden el aprendizaje, la necesidad de estructurar nuevas teorías, enfoques y paradigmas que orienten e incidan, desde una visión más científica y real, las prácticas pedagógicas a la luz de los nuevos tiempos, tal es el caso de la sicología, la epistemología en la filosofía y recientemente la neurociencia.

Durante décadas, el intento por la comprender la mente, el pensamiento y el aprendizaje, ha representado una ardua búsqueda, debido en parte a la falta de herramientas interdisciplinarias poderosas para la investigación y la generación de conocimiento actualizado que respalde decisiones educativas. Sin embargo, el mundo de hoy nos sitúa ante una extraordinaria producción de estudios científicos sobre la mente y el cerebro, sobre los procesos de pensamiento y su influencia en el aprendizaje y sobre la vasta gama de procesos neuronales que ocurren durante el pensamiento 
y el aprendizaje. Esta dinámica revolución que ha ocurrido en el estudio de la mente durante los últimos años tiene importantes implicaciones en la pedagogía contemporánea y, consecuentemente, en la formación de los futuros pedagogos.

Hoy, gracias a los aportes de recientes estudios neurológicos, sabemos que obedecemos a estímulos internos y externos debido a nuestra condición humana, lo que nos lleva a cuestionar: cuáles son estos estímulos y de qué forma intervienen en el aprendizaje y la génesis de conocimiento personal de cada individuo.

El trabajo científico ha abarcado una amplia gama de temas de la cognición y la neurociencia en el aprendizaje, la memoria, el lenguaje y el desarrollo cognitivo. A medida que los científicos continúan estudiando el aprendizaje, emergen nuevos lineamientos, paradigmas y metodologías de investigación, los cuales, probablemente, modifiquen las actuales concepciones teóricas. Esto, debido a que en los últimos años ha aumentado notablemente el interés de la neurociencia por estudiar la capacidad cognitiva en todas las edades de los seres humanos.

Numerosos estudios han demostrado que las aseveraciones sobre las etapas del desarrollo humano establecidas en el pasado no son tan universales como cabría esperarse, por lo que se estima que el conocimiento alcanzado, en relación con nuestras capacidades cognitivas, constituye un conocimiento inacabado en continua transformación. Gracias a la incursión de dichos estudios, como señala Ratey (2003), se advierte:

Aprendemos a lo largo de la vida adulta ya que nuestros cerebros son maravillosamente plásticos...nuestra estructura cerebral no está predeterminada o fijada. Podemos alterar el desarrollo en marcha de nuestros cerebros y por lo tanto, nuestras capacidades. (p. 34)

Esta afirmación revalida la idea de precisar el aprendizaje como un fenómeno en el que se fusionan múltiples procesos mentales a partir de la interacción de las personas con su entorno social, en el que se generan aprendizajes en diversos matices que van desde el aprendizaje espontáneo y orgánico, al aprendizaje estimulado y orientado, tal es el caso del amplio espectro de aprendizajes que se gestan en el entorno escolar.

El aprendizaje es un proceso inherente al ser humano y se da a lo largo de la vida. Por lo tanto, no requiere necesariamente de la intervención "intencionada" de otra persona para que ocurra. Este sentido del aprendizaje continuo se ha ignorado, ya que se asume, por defecto, que el aprender solo se da en entornos escolares con la agravante de la existencia de un rezago significativo, si consideramos el avance de otras actividades del quehacer humano, tal es el caso de la enseñanza formal. En la pedagogía contemporánea es necesario comprender y consensar que el aprendizaje de todo individuo es continuo y permanente, y que la vida y la mente del niño y la niña, desde la pedagogía, así lo requieren.

Quizá el sitio ideal para observar y analizar el fantástico fenómeno del aprendizaje humano es, en sí misma, la escuela; ya que por su carácter formativo constituye el espacio donde converge necesariamente el pensamiento, el conocimiento, así como una fuerte y rica interacción social. Lo anterior nos hace reflexionar acerca de cuál metodología es la ideal y la que mejor se ajusta a las formas de comunicación que utilizamos para relacionarnos con el entorno físico y social, ya que el mismo lenguaje ejerce un papel insustituible en la percepción e interpretación del mundo y sobre todo en la forma de cómo aprendemos y el valor que le damos a lo aprendido, la invención y uso de nuevos códigos comunicativos y su influencia en el pensamiento.

Desde esta perspectiva, John Ratey, al afirmar que "la memoria es la fuerza centrípeta que junta el aprendizaje y la conciencia” (2003, p. 230), aporta información valiosa concerniente al 
inestimable papel de la memoria, la cual, desde diversos enfoques tradicionalistas del aprendizaje, ha sido lamentablemente satanizada como mecanismo que niega y excluye el aprendizaje significativo. Sin embargo, la memoria, más que un proceso lineal de recordar y hacer, se comporta como un sistema complejo de procesos que dependen, en gran medida, del material que se debe memorizar y de la forma en que este se presenta al sujeto que aprende. Se plantea una nueva visión del aprendizaje: mucha parte del aprendizaje y de la adquisición de nuevos conocimientos dependen, en gran medida, de dónde y de qué manera fijamos e interiorizamos la información y los nuevos conocimientos. Al respecto Ratey (2003) señala:

La formación y la recuperación de cada recuerdo están influidas por el estado del ánimo, el entorno por configuraciones psicológicas presentes en el momento en que se forma o recupera el recuerdo. Por eso recordarán de maneras distintas diferentes personas un mismo hecho. No hay por necesidad una persona que "tenga razón”, no está por necesidad las demás “equivocadas". La memoria cambia además a medida que nosotros nos transformamos con el tiempo. Nuestras experiencias modifican nuestras actitudes, y por lo tal cómo y qué recordamos. (p. 231)

Las implicaciones directas de esta afirmación nos sitúan ante un vasto panorama de nuevas posibilidades que podrían descifrar la infinidad de procesos mentales que se llevan a cabo antes, durante y después del aprendizaje. A partir de esta idea, resulta indispensable dedicar una mirada a la formación y actitudes de los docentes y las docentes, a la metodología que utilizarán y a las distintas formas de interacción posible que podrían generar en cualquier contexto educativo, ya sea desde la visión formal o no formal.

No podemos negar que, debido a nuestra condición humana, obedecemos a estímulos; pero, cuáles son estos estímulos y de qué forma intervienen en el aprendizaje y la génesis de conocimiento personal de cada individuo y, consecuentemente, en las metodologías que deben atender integral y eficazmente tal diversidad. Se hace necesario, por lo tanto, dirigir la atención a la generación de nuevas teorías conducentes a novedosas concepciones del diseño curricular, la enseñanza, el lenguaje como herramienta para el aprendizaje y el aprendizaje mismo. Teorías muy diferentes de las que comúnmente se encuentran en las instituciones educativas en la actualidad. Igualmente importante es comprender y asimilar el incremento de investigaciones interdisciplinarias y nuevos aportes científicos que, desde la neurociencia, han comenzado a hacer de cierta manera más visible el camino que conduce de la investigación profunda y justa de la práctica pedagógica en la actual coyuntura educativa.

En el ámbito educativo, este proceso se expresa en la configuración de un nuevo paradigma que nos obliga a concebir el aprendizaje como el proceso mediante el cual cada persona construye la plataforma necesaria para apropiarse de los conocimientos, las habilidades, las destrezas y los valores que requiere para un desarrollo integral como ser humano.

En este nuevo paradigma educativo, cada persona es ahora co y autoconstructora de su propio conocimiento, más que consumidora del mismo. Por lo tanto, la acción docente se debe reconfigurar en la de guía del estudiante en el proceso que este ha de llevar a cabo para construir su conocimiento, como algo nuevo. En esta génesis del conocimiento inicial, el mismo es enriquecido o transformado significativamente durante el proceso. Esta perspectiva del conocimiento está en contraposición con la visión más tradicional del mismo, consistente en un proceso de transmisión o adquisición de «parcelas» o «trozos» de conocimiento, lo que ha llevado a una concepción errada y simplificada de procesos altamente complejos. 
Gracias al sorprendente avance de la neurociencia, estamos llegando a descifrar de manera más precisa, cómo se da el proceso de desarrollo cerebral y la trascendencia de la información sensorial que se origina de las experiencias directas y multisensoriales para la construcción del conocimiento. Al respecto Hospers (1976) señala:

(...) entre todas las vías o fuentes del conocimiento, la experiencia sensorial es la más obvia. Ciertamente parece que podemos saber mucho más cosas del mundo -que existen cosas físicas y cuáles son sus características- mirando, oyendo, tocando, oliendo, gustando. Es primariamente a través de la vista y el tacto por lo que sabemos que existen cosas físicas... (p. 160)

Al respecto, muchos estudios han demostrado que existen diferentes vías o fuentes para construir conocimiento y diferentes vías para expresarlo y aplicarlo. Si por encima de estas vías generamos herramientas pedagógicas componentes que atiendan estilos diferenciados y propios de aprendizaje, de creatividad, de expresión, de música, de movimiento, solo por mencionar algunas, hallaremos un sin fin de oportunidades para gestar una propuesta de aprendizaje que atienda de manera estimulante y diversificada a cada persona. Entre las virtudes más sobresalientes de estos mecanismos, lo primordial lo constituye la concepción de un entorno idealmente estimulante para un cerebro que tiene ansia por aprender, pues las experiencias directas y concretas mediante metodología activa estimulan el desarrollo de los sistemas perceptivos sensoriales, de los sistemas motores, de diferentes regiones en el cerebro y la consolidación del conocimiento vivido. Desde esta óptica, no podemos obviar el gran peso que ejerce, además, la razón como fuente de conocimiento, de acuerdo con lo señalado por Hospers (1976):

(...) el significado de la palabra razón no se agota, y se supone que es la razón lo que estamos considerando fuente de conocimiento. Consideremos este sentido más amplio. El razonamiento es algo que hacemos; pero la razón es una capacidad. La razón es la capacidad de pensar, y el grado de nuestros poderes racionales, o poderes de razón, es el grado de nuestra capacidad para pensar (...) si no pudiéramos pensar, no podríamos adquirir ningún conocimiento; el tener esta capacidad es indispensable para obtener conocimientos de cualquier clase, incluso el conocimiento adquirido mediante percepción sensorial. (p. 173)

Cada individuo aprende de forma única y desarrolla una relación particular, exclusiva e irrepetible con el conocimiento, permeada de sus vivencias y de su interacción con su espacio físico y social, lo que nos hace reflexionar acerca de la trascendencia del lenguaje y las formas de comunicación que utilizamos para relacionarnos con el entorno.

Definitivamente, el entorno ejerce un papel insustituible en la percepción e interpretación del mundo y, sobre todo, en la forma de cómo aprendemos y valoramos lo aprendido, la invención y uso de nuevos códigos comunicativos y su influencia en el pensamiento. De esta forma, la cultura, como elemento innato y presente de cualquier sociedad, interviene con gran fuerza en la lengua de los grupos humanos, ya que la misma se ve influenciada y evoluciona vertiginosamente de acuerdo con el uso que se le dé, con los valores, saberes y conocimiento que han sido construidos y compartidos socialmente por sus integrantes.

Es necesario que comprendamos que las "clásicas" teorías del aprendizaje han "intentando" explicar de qué forma aprendemos los seres humanos. Desafortunadamente, han persistido a lo largo de los tiempos, posiciones extremas y opuestas entre sí, que han negado toda posibilidad de encuentro y complementariedad entre estas teorías, dejando serios vacíos y una profunda huella 
no solo en la formación de docentes, sino además y por consecuencia, en todos los niveles de la educación y en términos universales.

Como prueba fidedigna de la búsqueda de nuevas explicaciones y posibilidades de profundización en este campo, actualmente la neurociencia y neurodidáctica nos compromete, en primera instancia, al principio epistemológico fundamental de que nuestra labor formadora, en cualquiera de sus modalidades, no debe fundamentarse únicamente en teorías anquilosadas, sino además nos conceden múltiples razones para abordar el acto educativo en concordancia con las nuevas condiciones que nos plantea esta compleja y nueva coyuntura educativa, más allá de posiciones tradicionalmente convenientes para unos, pero injustas para otros....

Al respecto han surgido importantes posiciones que sugieren la noción sobre la particularidad de cada ser humano en relación con su propio aprendizaje y el conocimiento. Entre estos aportes orientadores de la búsqueda de la comprensión del aprendizaje, tenemos que considerar las valiosas contribuciones de Gardner, Hospers, Brizendine, Campbel, Ratey, entre otros, quienes han demostrado a la pedagogía, a la didáctica y a la sicología, que las personas son seres únicos y con cerebros que aprenden por diversas y múltiples vías y en estas se debe aprovechar la curiosidad de la persona por aprender. Esta y otras nuevas posiciones epistemológicas y biopedagógicas deberían permear a nuestros futuros docentes, pues desde la formación misma de los docentes se debe instituir la utilización de nuevos y diversificados elementos que permitan a sus estudiantes, en su ejercicio profesional: explorar, aprender, apropiarse del conocimiento pedagógico de punta, asimismo, estimular el placer por las propuestas del aprendizaje de cualquier tipo siempre y cuando se orienten al descubrimiento y al asombro por aprender. Desde esta óptica, nada parece más razonable que concluir que la construcción de esta plataforma del conocimiento pedagógico debiera constituirse en un proceso indispensable en la formación de todo educador y educadora.

\section{Un actualizado y enriquecido conocimiento pedagógico: condición ideal para el aprendizaje}

En el quehacer de la práctica educativa, el éxito del aprendizaje empieza con la motivación de la persona por aprender. Este entusiasmo o motivación detona, en el cerebro, un sistema casi ininterrumpido de funciones que van desde la percepción sensorial hasta la evocación del aprendizaje ya interiorizado e integrado funcionalmente. Iniciar esta aventura de construcción de conocimiento pedagógico e ingeniería docente equivale a desprenderse de todo prejuicio y visión tradicional de la educación, así como a reordenar nuestras prioridades e ideas y permearnos de valiosos saberes provenientes de otros campos de estudio como lo son la sicología, la filosofía y otras disciplinas que traspasan las fronteras del orden cognitivo. No se trata solamente de una transformación pedagógica, sino de una redefinición del rol del docente y la docente como pedagogos y pedagogas, en donde deben manifestar, más que nunca, su papel insustituible como generadores de espacios y procesos orientados a la producción constante y efectiva de conocimiento en sus estudiantes. Este rasgo en el perfil del nuevo docente constituye un ingrediente esencial para propiciar la activación de la formación docente, inspirando posiciones metodológicas que gesten clases poderosas y promuevan, en los jóvenes docentes, estilos dinámicos de enseñanza; al tiempo que contribuyan a compartir reflexiones, decisiones, interrogantes y propuestas. Para que, así, disfruten y apliquen creativamente sus propios conocimientos a partir del ejercicio pedagógico.

Con un mayor conocimiento, basado primero en la experiencia acerca de las demandas reales de aprendizaje de estudiantes-docentes, unido al desarrollo de la sensibilidad hacia la 
forma más adecuada que atienda la complejidad del aprendizaje, el docente de formadores, por su carácter inspirador, debe brindar una formación personalizada y una atención más respetuosa de las diversidades individuales. El propósito de ello es facilitar el acceso a los contenidos desde las diferencias inherentes a cada persona y, también, fomentar actitudes cooperativas y de mayor integración social, ya que como espacio para el modelaje, el aula universitaria puede inculcar actitudes y saberes pedagógicos respaldados en los nuevos aportes de la neurociencia y neurodidáctica.

Desde esta visión, el papel del formador de formadores es imprescindible para la incorporación de estrategias inspiradas en los aportes de la neurociencia y que deberían aplicarse en el ámbito escolar. Su capacidad, creatividad, responsabilidad y sus altos conocimientos deben ser sus principales recursos para la transformación en la práctica pedagógica de los futuros docentes. De igual manera los conocimientos aportados desde los estudios de neurociencia y que paulatinamente han de adquirir, en torno a temas científicos facilitarán la comprensión del aprendizaje más allá de lo tradicionalmente aceptado desde la pedagogía.

Otro componente de la formación docente es el que se relaciona con los procesos evaluativos que han constituido, en los últimos años, ejes fundamentales sobre los que ha versado buena parte de las discusiones, debates y decisiones en el ámbito de las ciencias de la educación. Si la evaluación resulta esencial en cualquier tipo de proceso formativo, en la formación de formadores resulta una prioridad. En este tipo de específico de formación es donde se debe permear, con prácticas metodológicas y evaluativas acertadas, la práctica pedagógica de quienes formamos. En nuestra realidad formadora, la tarea de evaluar, en muchos casos, se nos presenta como una de las que implican mayor dificultad desde la perspectiva del formador de formadores. En nuestra labor docente, esta tarea puede presentar, en ocasiones, serias incongruencias de orden práctico ante determinados procesos de enseñanza-aprendizaje, como el caso de aquellas acciones pedagógicas innovadoras lamentablemente opacadas por las prácticas evaluativas convencionales que han sido inscritas en todos los niveles de la educación

El modo de evaluar, de realizar la devolución de las evidencias de lo aprendido, debe concebirse, como norma general, en un espacio común de participación al que tengan acceso todo el estudiantado. La tarea de valoración de los procesos que se gesten en el espacio áulico debe ser compartida, lo cual es imprescindible si se desea incidir integralmente en la formación de los futuros docentes quienes deberán incorporar, en su práctica profesional, criterios de corrección y de evaluación concordantes con los nuevos postulados pedagógicos y a la luz de la dinámica y compleja sociedad del conocimiento. Esta coyuntura nos exige fomentar en los jóvenes docentes, no solo la aplicación de renovados modelos y técnicas de evaluación, sino además la adaptación y transferencia de tales modelos evaluativos a las diversas condiciones y contextos con particularidades de diversidad de toda índole. Si uno de los mandatos de la nueva pedagogía se refiere a la contextualización de los contenidos y metodología en función del aprendiz, con mucho mayor razón se debe insistir en visualizar dicho tratamiento en las prácticas evaluativas.

Con base en todo lo anteriormente expuesto, se reafirma la gran influencia que ejerce el formador de formadores en el futuro ejercicio de la docencia de quienes estarán en las aulas en un futuro cercano. Deben vislumbrar que las diversificaciones de los métodos y modelos de enseñanza que desarrollen puedan incidir positivamente en la cimentación del conocimiento pedagógico. Tal renovación debe orientarse hacia una disposición al cambio desde la práctica pedagógica, basada en un profundo estudio y análisis científico de los procesos cognitivos que se desencadenan en el hábitat escolar. En un amplio espectro que va desde las bases biológicas del que aprende, hasta la naturaleza social de su entorno. Este tipo de noción o comprensión no es meramente técnica, ni exclusivamente reflexiva. No es sólo el conocimiento de los contenidos, ni el dominio genérico de la 
metodología y la didáctica. Es sumamente complejo porque debe intervenir, además, la experiencia y la intuición del docente o la docente.

Esta coyuntura educativa constituye una oportunidad para reflexionar e intentar comprender lo que acontece o no acontece en el aula escolar, lo que ocurre en las mentes de quienes aprenden, y observar los avances en el campo de la neurodidáctica y dilucidar un nuevo horizonte en nuestra práctica pedagógica. Los cambios que está experimentado y experimentará "la escuela" y la sociedad, no solo deben ser precisados y atendidos por los nuevos pedagogos, sino que deben constituirse en la materia prima para la sensibilización y disposición al cambio desde los formadores de docentes. De esta forma, se detonarán procesos de investigación y generación de propuestas pedagógicas en concordancia con la nueva sociedad costarricense y enmarcadas en una nueva era del conocimiento.

\section{Referencias bibliográficas}

Coronado, L., Goñi, A., Meza, J., Vargas M., Francis, S. (2009). Síntesis del Análisis de Problemas y Retos de la Formación Docente en el marco de la pedagogía actual. [Curso: Actualización Pedagógica de Formadores de las Carreras de Educación de las Universidades Estatales]. Recuperado el 7 de mayo de 2010, de http://campusvirtual.uned.ac.cr/lms/mod/resource/view. php?id $=5860$

Hospers, J. (1976). Introducción al análisis filosófico. Barcelona: Alianza.

Latapí, P. (2003). ¿Cómo aprenden los maestros? [Conferencia Magistral en el XXXV aniversario de la Escuela Normal Superior del Estado de México. Toluca, 18 de enero de 2003. En Secretaría de Educación Pública de México. (2003)] . Cuadernos de Discusión 6, 1-28. México: Secretaría de Educación

Pichardo, M. C., García, A. B., De la Fuente, J. y Justicia, F. (2007). El estudio de las expectativas en la universidad: análisis de trabajos empíricos y futuras líneas de investigación. Revista Electrónica de Investigación Educativa, 9(1). Consultado de http://redie.uabc.mx/vol9nol/ contenido-pichardo.html

Ratey, J. (2003). El cerebro. Manual de instrucciones. Barcelona: Random House. 